\title{
ESTUDIO SEDIMENTOLOGICO DE LA RAMBLA DE CHIVA
}

\author{
E. Sanjaume* \\ F. Segura* \\ M.J. Meyer*
}

\begin{abstract}
RESUMEN
Este artículo pretende estudiar los procesos actuales que se producen en la Rambla de Chiva (Valencia), mediante el análisis de sus sedimentos. Los datos obtenidos han permitido reconstruir la circulación del agua en la última crecida. Asimismo, se ha intentado caracterizar las diferencias existentes entre las barras y los canales de la sección analizada, asi como de las microformas que en ella se establecen.
\end{abstract}

\section{SUMMARY}

In this paper we have studied the present processes ocurring at the Rambla de Chiva (Valencia), by means of the sediment granumetical analysis. The data so obtained allowed us to establish the water flow in the last flood. We have also tried to determine the differences between the bars and channels as the studied section, as well as the microforms located on them.

La elección de esta rambla para el presente estudio obedece a su carácter autóctono y al tamaño asequible de su cuenca, ubicada entre dos de las grandes arterias que drenan el País Valenciano: el Turia y el Xúquer. La Rambla de Chiva nace de la confluencia del Barranco del Gallo y del Barranco Grande. A partir de su paso por el término de Cheste, cambia su denominación por la de Rambla de Poyo y con este nombre prosigue hasta la desaparición de su cauce en las inmediaciones del término municipal de Manises. Sin embargo, sus aguas circulan siguiendo la línea de máxima pen-

* Departamento de Geografía. Universidad de Valencia. 


\section{E. SANJAUME, F. SEGURA, M.J. MEYER}

diente y enlazan eventualmente con el Barranc de Torrent, por el cual desembocan en la Albufera de Valencia. Su último tramo - totalmente arfificializado- se ha convertido en una de las múltiples acequias que drenan la marjal de la Albufera (fig. 1).

Ante la diversidad de nombres con que se conoce este curso fluvial (Rambla de Chiva, Rambla de Poyo, Barranc de Torrent), se ha optado por la primera denominación, que ya fue utilizada en 1893 por VILANOVA y PIERA, aunque el muestreo se efectuó en el tramo intermedio del sector conocido como Rambla de Poyo.

El presente estudio intenta un análisis textural de los distintos microambientes que se han formado como resultado de los procesos actuales que operan en la citada rambla. Como es obvio, no se pretende en absoluto que los resultados aquí obtenidos puedan extrapolarse a todas las ramblas valencianas. Para poder caracterizar de un modo totalmente fiable la textura de cada uno de los microambientes de una rambla, serán necesarios todavía muchos más trabajos de investigación en este campo. Su síntesis permitirá, finalmente, llegar a establecer un modelo general.

\section{Definición de rambla}

Desde un punto de vista geomorfológico, una rambla puede definirse como un lecho fluvial que consta de dos secciones: una alta, similar a los canales de escorrentía perenne — con fuertes pendientes y poca anchura-, y otra baja, en la que se incrementa la anchura, aunque ésta sufre frecuentes oscilaciones que se relacionan con la textura del material del lecho y con la pendiente (THORNES, 1976, p. 36). En la cuenca alta se da un bajo índice de relación anchura/profundidad, y un alto grado de unión entre el canal y las vertientes adyacentes. En la cuenca baja, por el contrario, el índice de relación anchura/profundidad es mucho más elevado. Además, debido a la abundancia de material de lecho aumentan las pérdidas por trasmisión y se incrementa la capacidad de almacenaje de agua subálvea (THORNES, 1980, p. 236).

Hidrológicamente nuestras ramblas son valles secos calizos afectados por cambios alogénicos y autogénicos (MATEU, 1982, p. 135). Estos últimos hacen referencia principalmente a la permeabilidad del roquedo que facilita el desarrollo de un drenaje subterráneo. Entre las causas alogénicas habría que distinguir, según GREGORY and WALLING (1973, pp. 387395), las siguientes: antiguos cañones submarinos; sobreimposición de un curso fluvial que pasa de una litología impermeable a otra permeable; cambios climáticos que favorecen el descenso de la precipitación y/o de la evapotranspiración; descenso del nivel del acuifero por: sobreexplotación, recesión de las vertientes y/o incisión del valle, y por elevación del continente y/o descenso del nivel del mar. 
La Rambla de Chiva, por su características geomorfológicas, se ajusta perfectamente a la definición de rambla dada anteriormente. Se puede considerar, asimismo, que se trata de un valle seco cuya circulación efímera parece que está relacionada con la permeabilidad de las calizas y los cambios climáticos.

\section{Criterios de muestreo}

Cada medio sedimentario puede caracterizarse de acuerdo con la textura de sus sedimentos. Los primeros investigadores que trabajaron en este campo pretendían, sobre todo, averiguar la relación de un determinado sedimento con la roca madre, el sistema de transporte y medio de sedimentación. Se intentaba caracterizar y distinguir entre los grandes medios sedimentarios: glacial, fluvial, marino, etc.

A medida que la sedimentología ha ido avanzando, se ha constatado la multivariedad de microambientes existentes en cada uno de los medios. Así pues, el muestreo ha de ser cuidadoso, puesto que no puede tratarse del mismo modo el material correspondiente a una barra de canal, a un canal activo, a un lóbulo (point bar), a la llanura de inundación, etc. Se abren, por tanto, dos vías de investigación: caracterizar el medio en su conjunto, o bien cada uno de sus distintos microambientes. La primera ya ha sido seguida por numerosos autores, por lo que el medio fluvial en su conjunto ha quedado perfectamente definido en contraposición al resto de los medios sedimentarios.

En el presente estudio se ha optado por la segunda línea de investigación. Para ello se han muestreado cada una de las microformas existentes en el cauce a lo largo de un corte transversal. Se ha elegido como zona de muestreo una sección que por su anchura permitiera la existencia de diversos microambientes y que no estuviera visiblemente alterada por acciones antrópicas. Las muestras se han tomado en superficie dado que se pretende estudiar los procesos actuales que intervienen en el modelado de la Rambla de Chiva.

\section{Descripción de microambientes}

Las barras son quizá los rasgos más característicos de los cursos braided. Se forman donde se deposita el material de arrastre al disminuir las fuerzas de tracción de la corriente. Las localizaciones óptimas para su ubicación son: el comienzo de una curva, donde se ensancha el canal o en las uniones de los canales. En los tres casos se produce una divergencia de flujo. Inmediatamente aguas abajo, la corriente converge de nuevo lo que provoca una incisión (CHURCH and JONES, 1982, p. 293). Una vez formadas, las 
barras interfieren el flujo del canal, obligándolo a dividirse y reunirse de nuevo - en un modelo de distribución trenzado (braided) — con canales más o menos desarrollados en uno o en ambos lados de la barra.

Han sido muchos los intentos de clasificación morfológica de las barras, sin que hasta el momento se haya encontrado una que tenga validez universal. Una de las más conocidas y aceptadas es la clasificación propuesta por SMITH (1974), que distingue los siguientes tipos: barras longitudinales - con dos subtipos de transición: crescentic y medial-, transversales, de point bar, y diagonales.

Las barras longitudinales se forman en el centro de canales relativamente anchos. Son convexas y alargadas. Según LEOPOLD, WOLMAN and MILLER (1964, p. 238), su formación se inicia cuando se deposita la fracción más gruesa de la carga al decaer el flujo. Sobre este núcleo o aguas abajo de él, se van agregando nuevos materiales de menor calibre a medida que disminuye la corriente. Otros autores, sin embargo, discuten la necesidad de la existencia previa de un núcleo. ASHMORE (1982), por ejemplo, ha comprobado en los tanques de experimentación que las barras pueden formarse incluso con sedimentos de un solo calibre. Al migrar, las barras desarrollan caras de avalancha - si el sedimento es fino- y superficies de bajo ángulo, si el material es de grano grueso. Los restantes tipos de barras no se describen puesto que no se han reconocido en el área estudiada.

Las barras pueden evolucionar de un tipo a otro hasta convertirse en diagoriales que son las que presentan mayor estabilidad. Existe una clara relación entre la descarga de agua, el calibre del sedimento transportado, el tipo de barra que se desarrolla y la estructura interna resultante. Sin embargo, en los sedimentos actuales es muy difícil verificar la correspondencia entre la estructura interna y la morfología de la barra por la falta de cohesión de los materiales.

La sección estudiada del cauce de la Rambla de Chiva está formada por tres barras longitudinales - la que se instala en la margen derecha podría considerarse de tipo medial, puesto que sus bordes están siendo erosionados por canales transversales - separadas por dos canales centrales, a los que hay que añadir otros dos laterales. Dentro de cada barra se generan, a su vez, pequeñas microformas que no hacen más que repetir la disposición general de los elementos del cauce. Es por ello que aparecen pequeñas barras - asimismo longitudinales- separadas por microcanales.

En la fig. 2 se detalla la planta de la sección estudiada y la localización de cada una de las muestras. Hay que destacar que la barra de la margen derecha se ha generado sobre unos conglomerados antiguos - presumiblemente pleistocenos - con abundante matriz arcillosa. Se encuentra a más de $80 \mathrm{~cm}$. por encima del punto más bajo del cauce. Esta circunstancia favorece la formación de canales transversales que siguen la línea de máxima pen- 


\section{SEDIMENTOLOGIA DE LA RAMBLA DE CHIVA}

diente. La margen izquierda del cauce no se ha muestreado puesto que se sospechaba que podría haber sufrido alguna alteración.

\section{Resultados de los análisis granulométricos}

Las muestras se han sometido a un proceso de tamizado en los calibres correspondientes entre -6 y $4 \varphi$. La escasa cantidad de fracción fina ha hecho innecesario su análisis por lo irrelevante de sus resultados que no podrían alterar la tendencia media de la muestra. Una vez obtenidos los porcentajes, se han hallado los correspondientes parámetros e índices, utilizando las formulaciones teóricas propuestas por FOLK and WARD (1957).

De acuerdo con los valores que ofrece el índice de clasificación, se puede diferenciar claramente entre los sedimentos correspondientes a las barras y a los canales. Los tres canales muestreados - uno lateral de $9 \mathrm{~m}$. de anchura (muestra 1), y dos centrales: uno de $16 \mathrm{~m}$. (muestra 6 ) y otro de $5 \mathrm{~m}$. (muestras 10 y 11 ) - presentan muchas analogías y ciertas discrepancias.

Las cuatro muestras son sumamente heterométricas, especialmente la 1 , 10 y 11 , con histogramas polimodales (fig. 3), curvas tendidas y escasa clasificación. La peor selección la ostenta la muestra 1 , dado que los materiales aportados por la rambla se añaden a los erosionados del conglomerado basal. La muestra 6 es algo más homométrica y su histograma se presenta bimodal (fig. 3). Esta circunstancia, unida a la menor cantidad de material en rodamiento y suspensión, hace suponer que este canal es el activo y que por él ha circulado el agua incluso en los momentos de aguas bajas, lo cual incrementaría la selección de sus materiales.

Los calibres medios para las muestras 6,10 y 11 son elevados $(-4,13$, $-4,52$ y $-3,05$ respectivamente), lo que indicaría elevada energía de flujo, mientras que descienden notablemente en la 1. En este caso hay que tener en cuenta la matriz fina de los conglomerados basales que alteran la media real. Las mezclas y remociones son frecuentes en todos los canales, como demuestran las repetidas inflexiones que presentan las curvas. Estas mezclas pueden obedecer a remoción del sustrato - como la muestra 1 - o a nuevos aportes después de cada crecida.

Las curvas se dibujan tendidas en todos los casos (fig. 4), de acuerdo con la heterometría del material, a excepción de la muestra 6 que es claramente parabólica. En este caso se comprueba que la acumulación ha sido forzada, con material poco evolucionado, pero mejor clasificado que en las anteriores. En la mayoría de los casos el lavado ha predominado sobre la decantación. Las diferencias texturales entre las muestras 10 y 11 , que se encuentran en el mismo canal, sugieren que las condiciones de flujo no han permanecido constantes ni en el espacio ni en el tiempo. 
Todo el resto de los sedimentos analizados pertenecen a barras de canal. Sin embargo, por los resultados obtenidos habría que distinguir entre las características de la barra lateral y central. La primera -de $2 \mathrm{~m}$. de ancho- se encuentra atravesada por varios pequeños canales tanto longitudinales como transversales. Todas las muestras de esta barra $(2,3$ y 4$)$, tienen en común la fuerte heterometría de los materiales - que se manifiesta en sus histogramas polimodales (fig. 3) - y la escasa clasificación. Sus curvas son tendidas con ligera tendencia parabólica y marcadas inflexiones (fig. 4), como también sucedía en los canales.

Las proporciones de sedimentos transportados en saltación y suspensión sugieren que la energía de flujo no era muy fuerte en el momento del depósito. Las peculiares características de la muestra 4, con una parte del sedimento - el procedente de la rambla - mal clasificado, y otra parte algo mejor seleccionada que proviene de la erosión del conglomerado basal, así como el hecho de que en ella predomine el lavado, en tanto que en el resto lo hace la acumulación, parecen indicar la importancia que en esta muestra ha tenido el transporte transversal que ha arrastrado sedimentos desde la parte más alta del conglomerado hasta la parte más baja del canal. Por otro lado, la fuerte proporción de materiales en suspensión (90\%), en cuanto que en el resto predomina la saltación, sugiere que la elevada pendiente de este canal ha provocado un incremento de la turbulencia, con la consiguiente puesta en suspensión de mayor cantidad de sedimentos.

La muestra 5 corresponde a una barra de reconstrucción, ubicada en uno de los canales centrales, al pie de los canales transversales descritos anteriormente. La disposición textural de sus sedimentos y, sobre todo, las marcadas inflexiones de su curva denotan que los materiales que la integran presentan distinta procedencia. Una parte deriva de la destrucción de la barra superior, en tanto que otra procede del propio cauce en el que se instala.

Por último, las muestras 7,8 y 9 , corresponden a una barra central de $19 \mathrm{~m}$. de anchura, aunque cada una de ellas pertenece a una distinta microforma. La primera muestra presenta un histograma bimodal (fig. 3). Esto indica que es algo más homométrica que las anteriores. Su curva es sigmoidal (fig. 4), lo que supone una acumulación por exceso de carga. Sin embargo, su asimetría - ligeramente negativa- parece sugerir que estos materiales han experimentado un cierto lavado con posterioridad al depósito. La clasificación continúa siendo escasa.

Las dos muestras restantes son heterométricas y polimodales. Sus curvas acumuladas son tendidas con frecuentes inflexiones que denotan la mezcla del material de la barra subyacente con el aportado por la última crecida. La modalidad de transporte predominante es la saltación, lo que supone escasa energía de flujo en el momento del transporte. Los valores de asimetría son muy escasos, por lo que parece que lavado y decantación están equi- 
librados. Hay que tener en cuenta que se trata de materiales de formas de tipo acumulativo (barras) que pueden haber experimentado un cierto lavado posterior, como prueba la existencia de microcanales (muestra 8) que suponen cierta erosión.

\section{Conclusiones}

De los análisis realizados en la Rambla de Chiva podrían deducirse las siguientes conclusiones:

- El material de la rambla es sumamente heterométrico.

- Los histogramas son, por tanto, polimodales y presentan las mismas características que los materiales analizados por FUMANAL, CARMONA y MEYER (1982, p. 106), en la Rambla de Cervera.

- La clasificación en todos los materiales es muy escasa, ya que los valores de dicho índice oscilan entre 0,73 y 2,64 que equivalen, según lo indicado por FOLK and WARD (1957), a sedimentos moderadamente y mal clasificados respectivamente.

- Las curvas acumuladas son tendidas o con ligera tendencia parabólica, de acuerdo con el grueso calibre del material, su escasa evolución y mediocre clasificación.

- Son frecuentes las inflexiones en las curvas, lo que sugiere la existencia de remociones dentro del mismo sedimento, provocadas por las sucesivas crecidas o por la remoción del material subyacente.

- En general, dado el calibre de los materiales transportados en suspensión, puede decirse que se trata de un medio de elevada energía.

- Con todo, de estos análisis se desprende que la energía de flujo es mayor en los canales que en las barras. Es sintomático que en la mayoría de ellos el punto de ruptura para el material transportado en suspensión se establezca en -2 .

- Se ha constatado también que el material de las barras suele tener un calibre medio sensiblemente inferior al de los canales, lo que no sucede en el tramo superior de la misma rambla.

- No todos los canales presentan el mismo comportamiento. Habría que distinguir entre los del cauce y los microcanales situados en la superficie de las barras.

- En el primer grupo habría que diferenciar, a su vez, entre el canal activo - entendiendo que se trata del que lleva agua durante más tiempoy el resto de los canales. En estos últimos las variaciones observadas en cuanto a tamaño medio e índice de asimetría, parecen sugerir que las con- 
diciones de flujo no permanecen constantes ni en el espacio ni en el tiempo. Por lo que respecta al canal activo, este denota la mayor homometría y mejor selección de todas las muestras analizadas (dentro de la elevada heterometría y escasa clasificación de los sedimentos de la Rambla de Chiva). Esto podría indicar que al circular el agua incluso en los momentos de baja energía, se incrementa la selección.

- Los canales sobre las barras son de tipo erosivo, ya que inician - aunque parcialmente- la destrucción de la barra sobre la que se ubican. Por las características de sus parámetros se deduce que existe un equilibrio entre la acumulación preexistente y el lavado más reciente. En algunos casos, se aprecia un ligero predominio de este último.

- Por lo que respecta a las barras, las de grandes dimensiones no parece que cambien de emplazamiento después de cada crecida. Como mucho, se erosionan sus márgenes y se alargan o se acortan después de cada una de ellas. Para modificar totalmente su emplazamiento debería darse una crecida de enormes proporciones, con energías de flujo elevadísimas y turbulencias muy fuertes.

- Sin embargo, después de cada crecida se modifica el emplazamiento de las microformas existentes en las barras: tanto de los microcanales como de las microbarras.

- Habría que destacar también la escasa proporción de arena que esta rambla presenta en superficie. Dichos sedimentos, por su propio calibre, percolan entre los cantos y las gravas.

- Por último, hay que hacer constar que las conclusiones aquí aportadas se refieren exclusivamente al tramo analizado y pueden cambiar aguas arriba o aguas abajo de la misma rambla. 


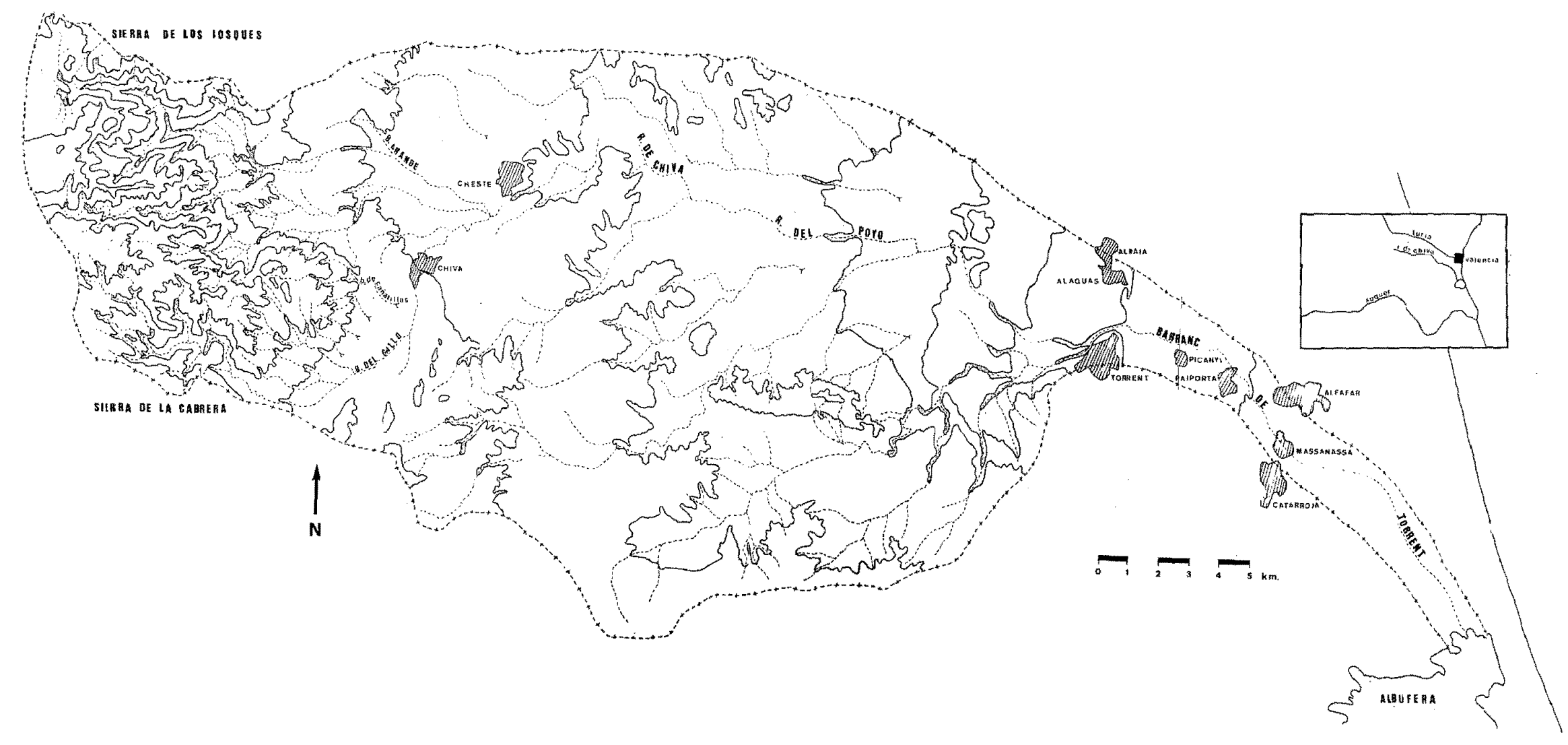




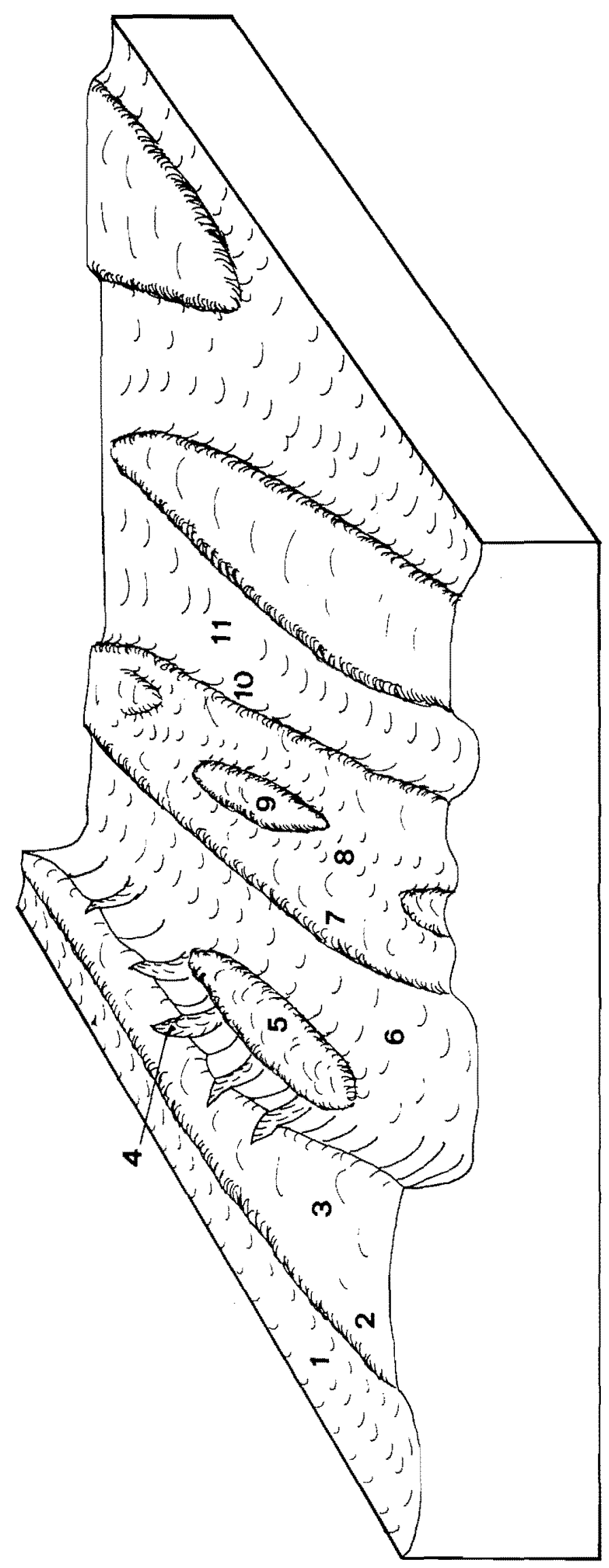

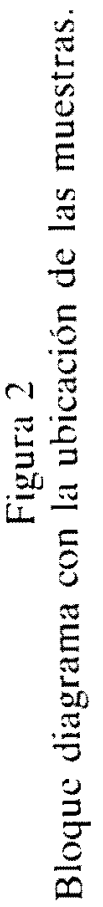


Figura 4

Curvas acumuladas.

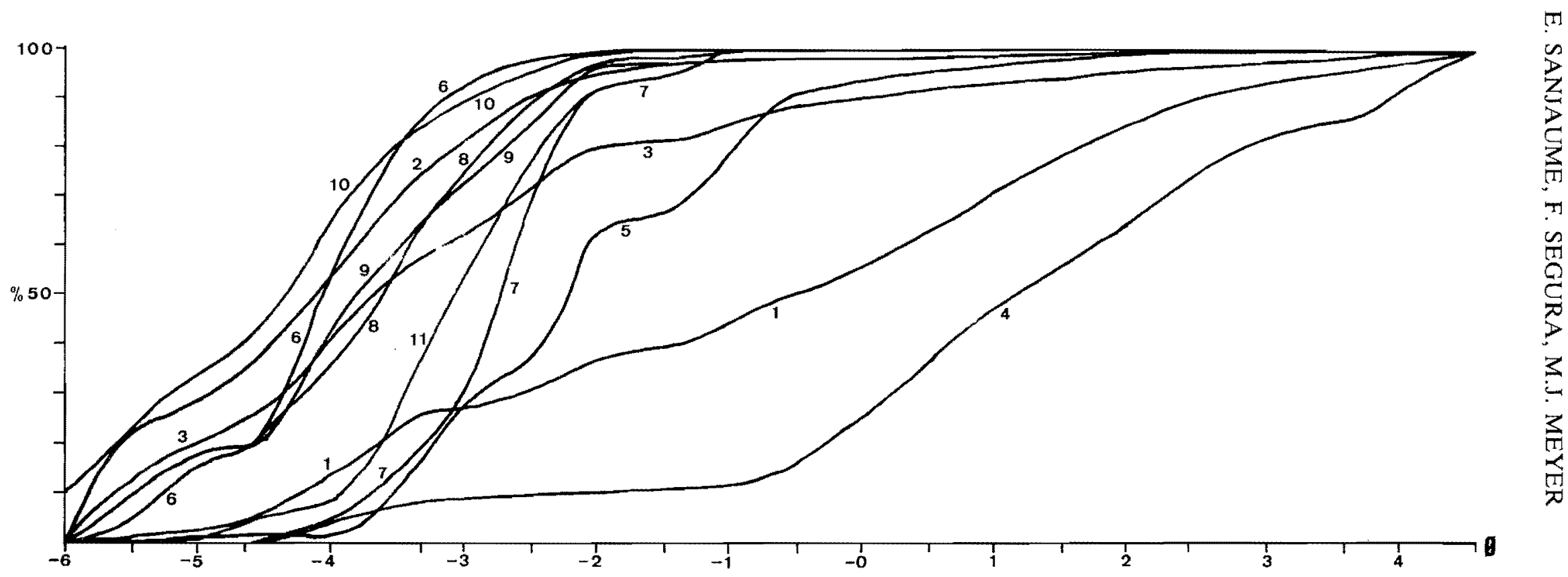




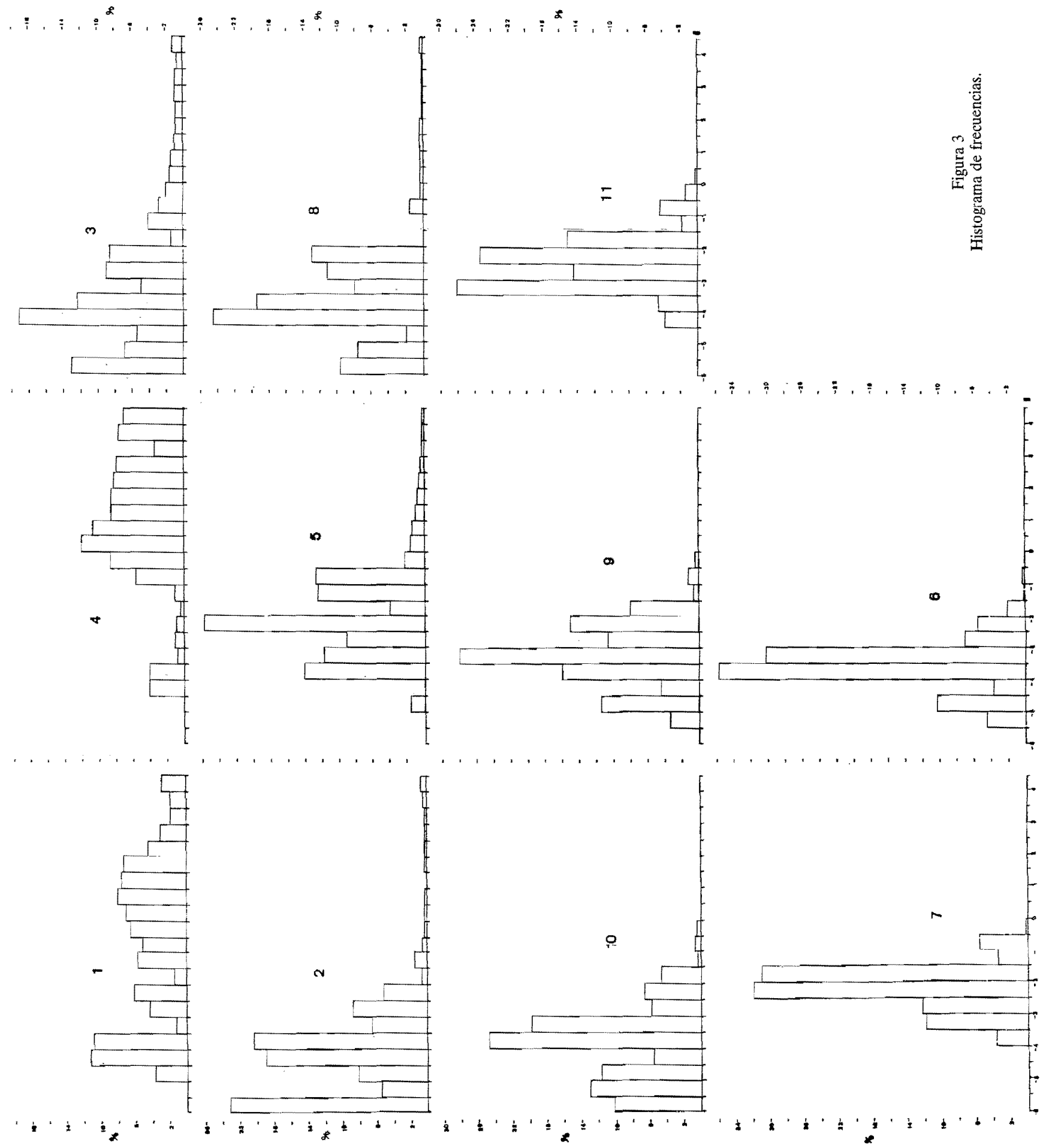




\section{Bibliografía}

ASHMORE, P.E. (1982): "Laboratory modelling of gravel braided stream morphology", Earth Surface Processes and Landforms, 7, pp. 201-225.

CHURCH, M.; JONES, D. (1982): "Channel bars in gravel-bed rivers", in THURST, J.C.; THORNE, C.R. (edit), Gravel-Bed River, Cf. pp. 291-338.

FOLK, R.L.; WARD, W.C. (1957): "Brazos River Bar: a study in the significance of grain size parameters", $J$. of Sed. Petrol., 27, pp. 3-26.

FUMANAL. M.P.; CARMONA, P.; MEYER, M.J. (1982): “Tres medios sedimentarios actuales en el Mediterráneo", Cuad. de Geogr., 31, pp. 97-128.

GREGORY, R.J.; WALLING, P.E. (1973): Drainage Basin: Form ans Process, London, E. Arnold, $458 \mathrm{pp}$.

LEOPOLD, L.B.; WOLMAN, M.G.; MILLER, J.P. (1964): Fluvial Process in Geomorphology, San Francisco, W.H. Freeman, 522 pp.

MATEU BELLES, J.F. (1982): El Norte del País Valenciano. Geomorfología litoral y prelitoral, Valencia, Universidad de Valencia, $286 \mathrm{pp}$.

SMITH, N.D. (1974): "Sedimentology and bar formation in the upper Kicking Horse River, a braided outwash stream", J. of Geol., 82, pp. 205-223.

THORNES, J.B. (1976): Semi-arid erosional systerns, London, School of Economics and Political Science, $79 \mathrm{pp}$.

THORNES, J.B. (1980): "Structural inestability and ephemeral channel behaviour", Z. Geomor. N.F., 36, pp. 233-244.

VILANOVA y PIERA, J. (1893): Memoria geognóstico-agricola y protohistórica de la provincia de Valencia, Madrid, Tip. Fortanet, $485 \mathrm{pp}$. 
